\title{
Facile preparation of novel $\beta$-substituted metalloporphyrins via Suzuki cross-coupling reaction
}

\author{
Rainer Gauler, Ralf Keuper, Andreas Winter*, and Nikolaus Risch \\ Department Chemie, Fakultät für Naturwissenschaften, Universität Paderborn, \\ Warburger Straße 100, D-33098 Paderborn, Germany \\ E-mail: $\underline{\text { awi@chemie.uni-paderborn.de }}$
}

Dedicated to Prof. Dr. Karsten Krohn on the occasion of his $60^{\text {th }}$ birthday

(received 21 Apr 04; accepted 13 Sept 04; published on the web 16 Sept 04)

\begin{abstract}
The Suzuki cross-coupling reaction of the porphyrin-zinc(II) complexes $\mathbf{1}$ and $\mathbf{2}$ and boronic ester 3a and various boronic acids 3b-l provided novel substituted porphyrins. These have been designed as building blocks in the synthesis of new photosensitizers for the photodynamic therapy (PDT) and for the preparation of porphyrin-containing supramolecular assemblies.
\end{abstract}

Keywords: Porphyrins, boronic acids, Suzuki cross-coupling, palladiumPd(0)-catalyzed C-C coupling

\section{Introduction}

The continuing interest in porphyrins and metalloporphyrins has encompassed areas in organometallic chemistry, ${ }^{1}$ material sciences, ${ }^{2}$ and photodynamic therapy (PDT). ${ }^{3}$ In the field of PDT research $\mathrm{C}-\mathrm{C}$ linked porphyrin-oligomers have been shown to be efficient photosensitizers in vivo, ${ }^{4-6}$ especially those possessing a substitution pattern related to that of the natural tetrapyrrole derivatives (e.g. hemato- or protoporphyrin). ${ }^{5,6}$

Palladium-mediated cross-coupling reactions of aryl halides with arylboronic acids (Suzuki coupling) are versatile methods for the synthesis of biaryls under mild reaction conditions. ${ }^{7}$ Furthermore, the Suzuki cross-coupling is largely unaffected by the presence of water, the reaction tolerates a broad range of functionalities, and yields non-toxic by-products. In spite of all these advantages only a few examples ${ }^{8}$ of this coupling reaction in the field of porphyrin synthesis have been described in the literature. 


\section{Results and Discussion}

To the best of our knowledge we describe the first application of the Suzuki reaction for the modification of such "natural porphyrins". ${ }^{9}$ The metalloporphyrins 1 (ZnBrDPDME, zinc(II)monobromodeuteroporphyrin dimethylester) and 2 ( $\mathrm{ZnBr}_{2} \mathrm{DPDME}$, zinc(II)-dibromodeuteroporphyrin dimethylester) easily obtainable from heme (inexpensive natural porphyrin source) $)^{10,11}$ were subjected to the reaction with boronic ester $\mathbf{3 a}$ and boronic acids $\mathbf{3 b} \mathbf{\mathbf { l }} \mathbf{\mathbf { I }}$ (Scheme 1).
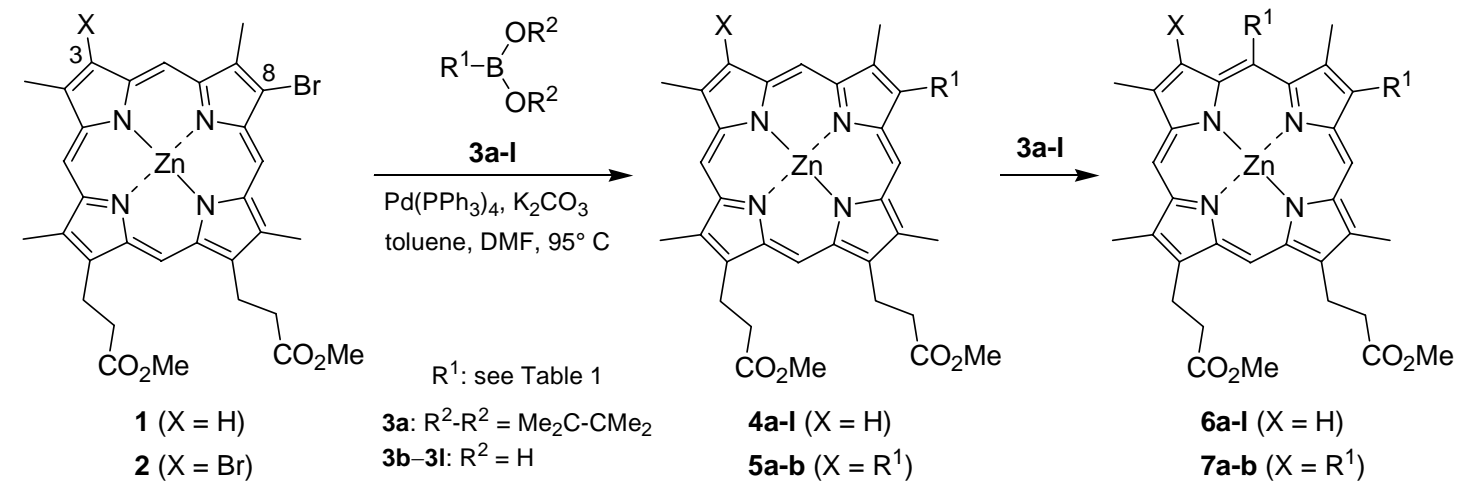

Scheme 1. Suzuki reaction of $\mathrm{ZnBrDPDME}$ (1) and $\mathrm{ZnBr}_{2} \mathrm{DPDME}$ (2).

The results of our experiments are summarized in Table 1. It is desirable to synthesize tetrapyrroles 4-7 with a great diversity of functional groups. Porphyrins linked by aryl spacers are the subject of many reports. ${ }^{11,12}$ In this connection $\mathbf{4 g , h , j}$ represent important key intermediates in the preparation of dimeric or oligomeric porphyrins by cross-coupling reactions.

Upon reaction of $\mathbf{1}$ with 2-allyl-4,4,5,5-tetramethyl-1,3,2-dioxaborolane 3a (entry 1, Table 1) a polar by-product was obtained, the porphyrin dimer 8 (Figure 1) as revealed by FAB mass spectrometry $\left(\mathrm{C}_{67} \mathrm{H}_{66} \mathrm{~N}_{8} \mathrm{O}_{8} \mathrm{Zn}_{2}\right.$ (1242.07); FAB-MS (NBA): $\mathrm{m} / \mathrm{z}$ (\%) 1238.32 (17) $\left[\mathrm{M}^{+},{ }^{64} \mathrm{Zn}\right]$ ). The unexpected formation of $\mathbf{8}$ can be explained by a Heck reaction of the Suzuki cross-coupling product 4a with boronic ester 3a. Remarkably, this reaction occurred using a stochiometric mixture of $\mathbf{1}$ and 3a indicating, that in this case the Heck reaction is much faster than the desired Suzuki cross-coupling reaction. Further investigations will show whether this method is suited for a highly efficient one-pot synthesis of coupled porphyrins.

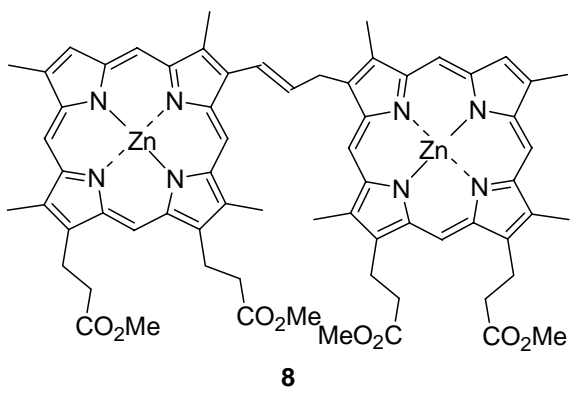

Figure 1. Dimeric metalloporphyrin 8 formed by Suzuki cross-coupling and Heck reactions. 
Table 1. Results of the Suzuki cross-coupling reaction of $\mathbf{1}$ and $\mathbf{2}$ with boronic ester $\mathbf{3 a}$ and boronic acids $\mathbf{3 b}-\mathbf{l}$

\begin{tabular}{|c|c|c|c|c|}
\hline Entry & $\begin{array}{l}\text { Metallo- } \\
\text { porphyrin }\end{array}$ & $\begin{array}{l}\text { Boronic acid } \mathbf{3}, \mathrm{R}^{1} \\
\left(\mathrm{R}^{2}=\mathrm{H} \text {, except } \mathbf{3 a}\right)\end{array}$ & $\begin{array}{l}\text { Reaction } \\
\text { conditions }\end{array}$ & $\begin{array}{l}\text { Product / } \\
\text { Yield }[\%]^{\mathrm{a}}\end{array}$ \\
\hline 1 & 1 & $3 \mathbf{a}^{\mathrm{b}}$ & $95^{\circ} \mathrm{C} / 15 \mathrm{~h}$ & $\mathbf{4 a} / 46$ \\
\hline 2 & 1 & $\mathbf{3 b},-\mathrm{CH}=\mathrm{CH}-\mathrm{C}_{5} \mathrm{H}_{11}$ & $95^{\circ} \mathrm{C} / 10 \mathrm{~h}$ & $\mathbf{4 b} / 79$ \\
\hline 3 & 1 & 3c, phenyl & $95^{\circ} \mathrm{C} / 15 \mathrm{~h}$ & $4 c / 60$ \\
\hline 4 & 1 & 3d, 1-naphthyl & $95^{\circ} \mathrm{C} / 14 \mathrm{~h}$ & $\begin{array}{l}\mathbf{4 d} / 46 \\
\mathbf{6 d} / 49\end{array}$ \\
\hline 5 & 1 & 3e, 3-nitrophenyl & $95^{\circ} \mathrm{C} / 48 \mathrm{~h}$ & $4 \mathbf{e} / 35$ \\
\hline 6 & 1 & 3f, 3-(trifluoromethyl)phenyl & $95^{\circ} \mathrm{C} / 48 \mathrm{~h}$ & $\begin{array}{l}\mathbf{4 f} / 55 \\
\mathbf{6 f} / 42\end{array}$ \\
\hline 7 & 1 & 3g. 3-bromophenyl & $95^{\circ} \mathrm{C} / 48 \mathrm{~h}$ & $\mathbf{4 g} / 21$ \\
\hline 8 & 1 & 3h, 4-bromophenyl & $95{ }^{\circ} \mathrm{C} / 48 \mathrm{~h}$ & $\mathbf{4 h} / 27$ \\
\hline 9 & 1 & 3i, 4-fluorophenyl & $95^{\circ} \mathrm{C} / 14 \mathrm{~h}$ & $\begin{array}{l}4 \mathbf{i} / 66 \\
\mathbf{6 i} / 32\end{array}$ \\
\hline 10 & 1 & 3j, 4-vinylphenyl & $95^{\circ} \mathrm{C} / 24 \mathrm{~h}$ & $\begin{array}{l}\mathbf{4 j} / 41 \\
\mathbf{6 j} / 35\end{array}$ \\
\hline 11 & 1 & 3k, 4-methoxyphenyl & $95^{\circ} \mathrm{C} / 18 \mathrm{~h}$ & $\mathbf{4 k} / 99$ \\
\hline 12 & 1 & & $95^{\circ} \mathrm{C} / 48 \mathrm{~h}$ & $-{ }^{c}$ \\
\hline 13 & 2 & 3j, 4-vinylphenyl & $95^{\circ} \mathrm{C} / 18 \mathrm{~h}$ & $\begin{array}{l}\mathbf{5 a} / 35 \\
\mathbf{7 a} / 28\end{array}$ \\
\hline 14 & 2 & 3k, 4-methoxyphenyl & $95^{\circ} \mathrm{C} / 15 \mathrm{~h}$ & $\begin{array}{l}\mathbf{5 b} / 68 \\
\mathbf{7 b} / 19\end{array}$ \\
\hline
\end{tabular}

${ }^{a}$ Isolated yield after flash column chromatography on $\mathrm{SiO}_{2}$ (eluent: $\mathrm{CH}_{2} \mathrm{Cl}_{2}$ ).

${ }^{\mathrm{b}}$ Boronic ester, 2-allyl-4,4,5,5-tetramethyl-1,3,2-dioxaborolane.

${ }^{\mathrm{c}}$ No 1,4-phenyl-tethered product could be isolated.

Using boronic acids 3d, 3f, 3i-k (Table 1, entries 4, 6, 9, 10, 13 and 14) in the reactions with 1 and 2 we were able to isolate compounds $\mathbf{6}$ and 7 from the crude product mixture by flash chromatography (Scheme 1). These products show greater $\mathrm{R}_{f}$ values in comparison with porphyrins 4 and 5. The ${ }^{1} \mathrm{H}$ NMR spectra of these compounds indicate unequivocally that one of the four possible meso-positions (positions 5, 10, 15 or 20) has been substituted by an aromatic substituent $\mathrm{R}^{1}$. Comparison with recently published experimental data (COSY NMR) leads us to 
suggest that position 5 has been substituted selectively. ${ }^{13}$ Obviously, substitution is only possible if the reactivity of the metalloporphyrins $\mathbf{1}$ and $\mathbf{2}$ is enhanced by a preceding substitution at the 3and/or 8-position ( $\beta$-pyrrole position). This observation was verified by the failure of the reaction of $\mathrm{Zn}(\mathrm{II})$-deuteroporphyrin dimethylester (ZnDPDME) and $\mathbf{3} \mathbf{j}$; in this case a $\mathrm{Pd}(0)$-catalyzed substitution occurred in neither position.

The direct formation of a phenyl-tethered bis(porphyrin) by using 4-(dihydroxyboryl)phenylboronic acid (31) failed (Table 1, entry 12). We isolated only a mixture of oligomeric porphyrinoids; the elucidation of their constitution is still under investigation.

With boronic acids $\mathbf{3 g}$ and $\mathbf{3 h}$ the yields of the expected products $\mathbf{4 g}$ and $\mathbf{4 h}$ are rather low (Table 1, entries 7 and 8), presumably due to a further Suzuki cross-coupling reaction of the bromophenyl-substituted metalloporphyrins. The appearance of small amounts of a 3-/8bromobiphenyl-substituted porphyrin supports this assumption.

\section{Experimental Section}

General Procedures. All reagents were purchased from commercial sources and used without prior purification unless specified. ZnBrDPDME (1) and ZnBr2DPDME (2) were obtained from heme as published previously. ${ }^{10,11}$ All solvents were dried and distilled according to standard procedures and stored under argon. Chromatographic separation was performed on silica gel (Merck, 0.04-0.063 mm). Melting points were obtained on a Büchi SMP-20 mp apparatus. NMR spectra were recorded on a Bruker ARX 200 instrument $\left({ }^{1} \mathrm{H}\right.$ at $200 \mathrm{MHz},{ }^{13} \mathrm{C}$ at $\left.50 \mathrm{MHz}\right)$ with TMS as internal standard. FAB mass spectrometry was carried out using a VG Autospec apparatus (glycerine/NBA/CsI-matrix, $30 \mathrm{keV}$ ). UV/Vis-spectra were measured on a Shimadzu 2101 spectrometer. Elemental analyses were obtained on a Perkin-Elmer M2400 analyzer.

\section{General procedure for Suzuki cross-coupling reaction}

The solution of the metalloporphyrin 1 or $2(0.21 \mathrm{mmol})$, potassium carbonate $(136 \mathrm{mg}$, $1.6 \mathrm{mmol})$, tetrakis(triphenylphosphine)palladium(0) (23 mg, $10 \mathrm{~mol} \%)$ and boronic acid ester 3a or a boronic acid 3b-l $(0.8 \mathrm{mmol})$ in a mixture of dry DMF $(5 \mathrm{~mL})$ and toluene $(5 \mathrm{~mL})$ was heated at $95{ }^{\circ} \mathrm{C}$ for $18 \mathrm{~h}$ in an atmosphere of argon. After cooling to room temperature water $(40 \mathrm{~mL})$ was added, and the solution was extracted with dichloromethane $(4 \times 50 \mathrm{~mL})$. The combined organic layers were washed with water $(3 \times 30 \mathrm{~mL})$, dried over sodium sulphate, and the solvent was removed under reduced pressure. Small amounts of DMF and toluene in the oily residue were removed under high vacuum. The resulting crude product, a brown solid was dissolved in a minimal volume of dichloromethane and purified by flash chromatography on silica gel (eluent: $\mathrm{CH}_{2} \mathrm{Cl}_{2}$ ) collecting fractions of approx. $10 \mathrm{~mL}$.

Zinc(II) 8-allyldeuteroporphyrin dimethylester (4a). According to the conditions of the General Procedure the reaction of metalloporphyrin 1 (136 mg, $0.21 \mathrm{mmol})$ and 2-allyl-4,4,5,5tetramethyl-1,3,2-dioxaborolane (3a) $(51 \mathrm{mg}, 0.3 \mathrm{mmol})$ resulted in the isolation of $4 \mathbf{a}(58 \mathrm{mg}$, 
$46 \%$ ) as a red-violet solid, mp $189-191{ }^{\circ} \mathrm{C}$ (dec.). ${ }^{1} \mathrm{H}$ NMR (200 MHz, $\left.\mathrm{CDCl}_{3}\right)$ : $\delta 3.01-3.12$ (m, $\left.4 \mathrm{H}, 13^{2} / 17^{2}-\mathrm{CH}_{2}\right), 3.25-3.54\left(\mathrm{~m}, 12 \mathrm{H}, \mathrm{CH}_{3, \text { pyr }}\right), 3.64-3.67\left(\mathrm{~m}, 6 \mathrm{H}, \mathrm{CO}_{2} \mathrm{CH}_{3}\right), 4.04-4.21$ (m, 4 $\left.\mathrm{H}, 13^{1} / 17^{1}-\mathrm{CH}_{2}\right), 4.41-4.59\left(\mathrm{~m}, 2 \mathrm{H}, 8^{1} / 3^{1}-\mathrm{CH}_{2}\right), 5.10-5.31\left(\mathrm{~m}, 2 \mathrm{H}, 8^{3} / 3^{3}-\mathrm{ABX}-\mathrm{CH}_{2, \text { vinyl }}\right), 6.36-$ $6.49\left(\mathrm{~m}, 1 \mathrm{H}, 8^{2} / 3^{2}-\mathrm{ABX}-\mathrm{CH}_{\text {,vinyl }}\right), 8.66,8.77$ (s, $\left.1 \mathrm{H}, 3(8)-\mathrm{CH}_{\beta-p y r}\right), 9.21-9.47$ (m, $\left.4 \mathrm{H}, \mathrm{H}_{\text {meso }}\right)$. ${ }^{13} \mathrm{C}$ NMR (50 MHz, $\left.\mathrm{CDCl}_{3}\right): \delta 11.36,11.42,11.64,11.74\left(\mathrm{q}, 2^{1} / 7^{1}, 12^{1}, 17^{1}-\mathrm{CH}_{3}\right), 13.65,13.68$ (q, $\left.7^{1} / 2^{1}-\mathrm{CH}_{3}\right), 21,85,21.98\left(\mathrm{t}, 13^{1} / 17^{1}-\mathrm{CH}_{2}\right), 30.61,30.89\left(\mathrm{t}, 3^{1} / 8^{1}-\mathrm{CH}_{2}\right), 37.34\left(\mathrm{t}, 13^{2} / 17^{2}-\mathrm{CH}_{2}\right)$, $52.12\left(\mathrm{q}, 13^{3} / 17^{3}-\mathrm{OCH}_{3}\right), 95.47,95.63,96.22,96.46,96.72,96.97,99.67$ (d, 5, 10, 15, 20-CH), $115.34,115.49\left(\mathrm{t}, 3^{3} / 8^{3}-\mathrm{CH}_{2}\right), 128.65,128.82(\mathrm{~d}, 8 / 3-\mathrm{CH}), 138.58,138.65\left(\mathrm{~d}, 8^{2} / 3^{2}-\mathrm{CH}\right), 135.94$, $136.01,136.12$, 136.20, 136.46, 136.62, 136.71, 136.82, 136.91, 137.72, 137.92, 138.11, 138.20, $139.43,139.43,139.64,140.02,145.65,145.71,145.83,145.95,146.17,146.54,146.66,146.92$, 147.05, 147.18, 147,28 (s, C quart), 174.11, $174.14\left(13^{3} / 17^{3}-\mathrm{CO}_{2} \mathrm{Me}\right)$. UV/VIS $\left(\mathrm{CH}_{2} \mathrm{Cl}_{2}\right): \lambda_{\max }(\mathrm{lg}$ ع) 567 (4.326), 531 (4.222), 402 (5.559), 326 (4.251). FAB-MS (NBA): m/z (\%) 640.12 (100) $\left[\mathrm{M}^{+},{ }^{64} \mathrm{Zn}\right]$. Anal. Calcd for $\mathrm{C}_{35} \mathrm{H}_{36} \mathrm{~N}_{4} \mathrm{O}_{4} \mathrm{Zn}(642.07) \mathrm{C}, 65.52 ; \mathrm{H}, 5.73 ; \mathrm{N}, 8.69$. Found $\mathrm{C}, 65.18$; H, 5.53; N, 8.39.

Zinc(II) 8-allyldeuteroporphyrin dimethylester (4b). Metalloporphyrin 1 (136 mg, $0.21 \mathrm{mmol})$ and boronic acid $\mathbf{3 b}(114 \mathrm{mg}, 0.8 \mathrm{mmol})$ afforded $\mathbf{4 b}(110 \mathrm{mg}, 79 \%)$ as a red-violet solid, mp 178-180 ${ }^{\circ} \mathrm{C}$ (dec.). ${ }^{1} \mathrm{H}$ NMR (200 MHz, $\left.\mathrm{CDCl}_{3}\right): \delta 1.13$ (t, $\left.3 \mathrm{H}, \mathrm{J}=7 \mathrm{~Hz}, 3^{7} / 8^{7}-\mathrm{CH}_{3}\right)$, 1.61-1.83 (m, $\left.4 \mathrm{H}, 3^{5} / 8^{5}, 3^{6} / 8^{6}-\mathrm{CH}_{2}\right) 1.88-1.95\left(\mathrm{~m}, 2 \mathrm{H}, 3^{4} / 8^{4}-\mathrm{CH}_{2}\right), 2.71-2.89\left(\mathrm{~m}, 2 \mathrm{H}, 3^{3} / 8^{3}-\right.$ $\left.\mathrm{CH}_{2}\right)$, 3.00-3.14 (m, $\left.4 \mathrm{H}, 13^{2} / 17^{2}-\mathrm{CH}_{2}\right), 3.31-3.55\left(\mathrm{~m}, 12 \mathrm{H}, \mathrm{CH}_{3, \mathrm{pyr}}\right), 3.64-3.66(\mathrm{~m}, 6 \mathrm{H}$, $\left.\mathrm{CO}_{2} \mathrm{CH}_{3}\right), 4.08-4.24\left(\mathrm{~m}, 4 \mathrm{H}, 13^{1} / 17^{1}-\mathrm{CH}_{2}\right), 6.62-6.86\left(\mathrm{~m}, 1 \mathrm{H}, 8^{2} / 3^{2}-\mathrm{CH}_{\text {vinyl }}\right), 7.61,7.70(\mathrm{~d}, 1 \mathrm{H}$, $\left.J=16.87 \mathrm{~Hz}, J=16.63 \mathrm{~Hz}, 3^{1} / 8^{1}-\mathrm{CH}_{\text {vinyl }}\right), 8.61,8.72\left(\mathrm{~s}, 1 \mathrm{H}, 8 / 3-\mathrm{CH}_{\beta-p y r}\right), 9.17-9.52(\mathrm{~m}, 4 \mathrm{H}$, $\left.\mathrm{H}_{\text {meso }}\right) .{ }^{13} \mathrm{C} \mathrm{NMR}\left(50 \mathrm{MHz}, \mathrm{CDCl}_{3}\right): \delta 11.40,11.49,11.67,11.90,12.49,12.59,13.54$ (q, $\mathrm{CH}_{3, \text { Pyr }}$ ), 14.87 (q, $\left.3^{7} / 8^{7}-\mathrm{CH}_{3}\right), 21.77\left(\mathrm{t}, 13^{1} / 17^{1}-\mathrm{CH}_{2}\right), 23.41$ (t, $\left.3^{6} / 8^{6}-\mathrm{CH}_{2}\right), 30.61\left(\mathrm{t}, 3^{5} / 8^{5}-\mathrm{CH}_{2}\right)$, $32.41\left(\mathrm{t}, 3^{4} / 8^{4}-\mathrm{CH}_{2}\right), 35.16,35.23\left(\mathrm{t}, 3^{3} / 8^{3}-\mathrm{CH}_{2}\right), 37.27\left(\mathrm{t}, 13^{2} / 17^{2}-\mathrm{CH}_{2}\right), 52.04\left(\mathrm{q}, 13^{3} / 17^{3}-\right.$ $\left.\mathrm{OCH}_{3}\right), 95.04,95.19,96.10,96.27,96.89,99.54\left(\mathrm{~d}, \mathrm{CH}_{\text {meso }}\right), 123.35,123.58\left(\mathrm{~d}, 3^{2} / 8^{2}-\mathrm{CH}_{\text {vinyl }}\right)$, $128.35,128.47$ (d, 8/3- $\left.\mathrm{CH}_{\beta-\text { pyr }}\right), 137.04,137.22$ (d, 81/3 $\left.{ }^{1}-\mathrm{CH}\right), 134.35,134.83,135.71,135.75$, $136.03,136.13,136,34,136.55,137.51,137.96,139.21,139.75,145.09,145.19,145.40,140.52$, $145.52,145.80,145.96 .146 .00,146.36,146.58,146.74,146.84,146.99,147.04$ ( $\mathrm{s}, \mathrm{C}_{\text {quart }}$ ), 174.06, $174.09\left(13^{3} / 17^{3}-\mathrm{CO}_{2} \mathrm{Me}\right)$. UV/VIS $\left(\mathrm{CH}_{2} \mathrm{Cl}_{2}\right): \lambda_{\max }(\lg \varepsilon) 572$ (4.028), 535 (3.903), 404 (5.051). FAB-MS (NBA): $\mathrm{m} / \mathrm{z}(\%) 696.14$ (23) $\left[\mathrm{M}^{+},{ }^{64} \mathrm{Zn}\right]$. Anal. Calcd for $\mathrm{C}_{39} \mathrm{H}_{44} \mathrm{~N}_{4} \mathrm{O}_{4} \mathrm{Zn}$ (698.18) C, 67.09; H, 6.35; N, 8.02. Found C, 66.78; H, 6.24; N, 8.07.

Zinc(II) 8-phenyldeuteroporphyrin dimethylester (4c). Metalloporphyrin 1 (136 mg, $0.21 \mathrm{mmol})$ and boronic acid $3 \mathbf{c}(98 \mathrm{mg}, 0.8 \mathrm{mmol})$ yielded $4 \mathbf{c}(81 \mathrm{mg}, 60 \%)$ as a red-violet solid, mp $186-187{ }^{\circ} \mathrm{C}$ (dec.). ${ }^{1} \mathrm{H}$ NMR (200 MHz, $\left.\mathrm{CDCl}_{3}\right): \delta 3.10-3.18(\mathrm{t}, 4 \mathrm{H}, J=7.72 \mathrm{~Hz}$, $13^{2} / 17^{2}-\mathrm{CH}_{2}$ ), 3.42-3.65 (m, $18 \mathrm{H}, \mathrm{CO}_{2} \mathrm{CH}_{3}, \mathrm{CH}_{3, \text { pyr }}$ ), 4.10-4.32 (m, $4 \mathrm{H}, 13^{1} / 17^{1}-\mathrm{CH}_{2}$ ), 7.76 (d, $\left.1 \mathrm{H}, J=7.46 \mathrm{~Hz}, \mathrm{CH}_{\text {aryl }}\right), 7.90\left(\mathrm{dd}, 2 \mathrm{H}, J=7.46, J=7.12 \mathrm{~Hz}, \mathrm{CH}_{\text {aryl }}\right), 8.20$ (d, $2 \mathrm{H}, J=7.12 \mathrm{~Hz}$, $\left.\mathrm{CH}_{\text {aryl }}\right), 8.96$ (s, $\left.1 \mathrm{H}, 8 / 3-\mathrm{H}_{\text {B-pyr }}\right), 9.58,9.74,9.89,9.97$ (s, $\left.4 \mathrm{H}, \mathrm{H}_{\text {meso }}\right) .{ }^{13} \mathrm{C} \mathrm{NMR} \mathrm{(50} \mathrm{MHz,}$ $\mathrm{CDCl}_{3}$ ): $\delta 11.64,11.66,13.99,14.22\left(\mathrm{q}, 2^{1}, 7^{1}, 12^{1}, 17^{1}-\mathrm{CH}_{3}\right), 21.72\left(\mathrm{t}, 13^{1} / 17^{1}-\mathrm{CH}_{2}\right), 37.15(\mathrm{t}$, $\left.13^{2} / 17^{2}-\mathrm{CH}_{2}\right), 52.06\left(\mathrm{q}, \mathrm{OCH}_{3}\right), 95.18,97.11,99.82,100.26(\mathrm{~d}, 5,10,15,20-\mathrm{CH}), 127.58$, $129.18,132.82\left(\mathrm{~d}, \mathrm{CH}_{\text {aryl }}\right), 129.06(\mathrm{~d}, 8(3)-\mathrm{CH}), 136.18,136.37,136.55,137.16,137.93,138.20$, 
$141.41,145.89,146.02,146.11,146.75,146.86,147.27,147.38,148.24$ (s, $\left.\mathrm{C}_{\text {quart }}\right), 174.02$ $\left(13^{3} / 17^{3}-\mathrm{CO}_{2} \mathrm{Me}\right)$. UV/VIS $\left(\mathrm{CH}_{2} \mathrm{Cl}_{2}\right): \lambda_{\max }(\lg \varepsilon) 570$ (4.316), 533 (4.235), 405 (5.325). FAB-MS (NBA): $\mathrm{m} / \mathrm{z}(\%) 676.08$ (29) $\left[\mathrm{M}^{+},{ }^{64} \mathrm{Zn}\right]$. Anal. Calcd for $\mathrm{C}_{38} \mathrm{H}_{36} \mathrm{~N}_{4} \mathrm{O}_{4} \mathrm{Zn}$ (678.11) C, 67.31; H, 5.35; N, 8.26. Found C, 66.98; H, 5.14; N, 8.05.

Zinc(II) 8-(naphth-1-yl)deuteroporphyrin dimethylester (4d) and Zinc(II) 5,8-di(naphth-1yl)deuteroporphyrin dimethylester (6d). Metalloporphyrin 1 (136 mg, $0.21 \mathrm{mmol}$ ) and boronic acid 3d (138 mg, $0.8 \mathrm{mmol})$ gave 4d (82 mg, 46\%) as a brown-violet solid, $\mathrm{mp} 197-201{ }^{\circ} \mathrm{C}$ (dec.) and $\mathbf{6 d}(100 \mathrm{mg}, 49 \%)$ as a brown-violet solid, $\mathrm{mp} 221-227^{\circ} \mathrm{C}$ (dec.).

4d. ${ }^{1} \mathrm{H}$ NMR $\left(200 \mathrm{MHz}, \mathrm{CDCl}_{3}\right)$ : $\delta 3.26-3.35\left(\mathrm{~m}, 4 \mathrm{H}, 13^{2} / 17^{2}-\mathrm{CH}_{2}\right), 3.49-3.82(\mathrm{~m}, 18 \mathrm{H}$, $\left.\mathrm{CO}_{2} \mathrm{CH}_{3}, \mathrm{CH}_{3, \text { pyr }}\right), 4.41-4.55\left(\mathrm{~m}, 4 \mathrm{H}, 13^{1} / 17^{1}-\mathrm{CH}_{2}\right), 7.36-7.46\left(\mathrm{~m}, 1 \mathrm{H}, \mathrm{CH}_{\text {aryl }}\right), 7.54-7.74(\mathrm{~m}, 1$ $\left.\mathrm{H}, \mathrm{CH}_{\text {aryl }}\right), 7.92-8.03$ (m, $\left.2 \mathrm{H}, \mathrm{CH}_{\text {aryl }}\right), 8.13-8.32$ (m, $\left.3 \mathrm{H}, \mathrm{CH}_{\text {aryl }}\right), 9.09$ (s, $\left.1 \mathrm{H}, 8 / 3-\mathrm{H}_{3-\mathrm{pyr}}\right), 9.85$, $10.07,10.15,10.33$ (s, $\left.4 \mathrm{H}, \mathrm{H}_{\text {meso }}\right)$.

6d. ${ }^{1} \mathrm{H}$ NMR $\left(200 \mathrm{MHz}, \mathrm{CDCl}_{3}\right): \delta 3.28\left(\mathrm{~s}, 3 \mathrm{H}, \mathrm{CH}_{3, \mathrm{pyr}}\right), 3.32-3.46\left(\mathrm{~m}, 7 \mathrm{H}, 13^{2} / 17^{2}-\mathrm{CH}_{2}\right.$, $\mathrm{CH}_{3 \text {,pyr }}$ ), 3.64 (s, $3 \mathrm{H}, \mathrm{CH}_{3 \text {,pyr }}$ ), 3.74-3.81 (m, $9 \mathrm{H}, \mathrm{CO}_{2} \mathrm{CH}_{3}, \mathrm{CH}_{3, \text { pyr }}$ ), 4.42-4.56 (m, $4 \mathrm{H}, 13^{1} / 17^{1}$ $\mathrm{CH}_{2}$ ), 7.20-8.35 (m, $\left.14 \mathrm{H}, \mathrm{CH}_{\text {aryl }}\right), 9.86,10.05,10.21,10.39$ (s, $4 \mathrm{H}, 8 / 3-\mathrm{H}_{\beta-p y r}, \mathrm{H}_{\text {meso }}$ ).

Zinc(II) 8-(3-nitrophenyl)deuteroporphyrin dimethylester (4e). Metalloporphyrin 1 (136 mg, $0.21 \mathrm{mmol})$ and boronic acid $3 \mathbf{e}(136 \mathrm{mg}, 0.8 \mathrm{mmol})$ furnished $4 \mathbf{e}(50 \mathrm{mg}, 35 \%)$ as a red-violet solid, mp 188-193 ${ }^{\circ} \mathrm{C}$ (dec.). ${ }^{1} \mathrm{H}$ NMR (200 MHz, $\left.\mathrm{CDCl}_{3}\right): \delta 3.12-3.19$ (m, $\left.4 \mathrm{H}, 13^{2} / 17^{2}-\mathrm{CH}_{2}\right)$, 3.39-3.61 (m, $\left.18 \mathrm{H}, \mathrm{CO}_{2} \mathrm{CH}_{3}, \mathrm{CH}_{3, \mathrm{pyr}}\right), 4.08-4.33$ (m, $\left.4 \mathrm{H}, 13^{1} / 17^{1}-\mathrm{CH}_{2}\right), 7.41-7.47$ (m, $1 \mathrm{H}$, $\left.\mathrm{CH}_{\text {aryl }}\right), 7.66-7.69$ (m, $\left.1 \mathrm{H}, \mathrm{CH}_{\text {aryl }}\right), 8.01-8.05$ (m, $\left.1 \mathrm{H}, \mathrm{CH}_{\text {aryl }}\right), 8.17$ (s, $\left.1 \mathrm{H}, \mathrm{CH}_{\text {aryl }}\right), 8.95$ (s, $1 \mathrm{H}$, 8/3- $\left.\mathrm{H}_{\beta-\text { pyr }}\right), 9.55,9.71,9.91,9.94\left(\mathrm{~s}, 4 \mathrm{H}, \mathrm{H}_{\text {meso }}\right)$.

Zinc(II) 8-(3-trifluoromethylphenyl)deuteroporphyrin dimethylester (4f) and zinc(II) 5,8di(3-trifluoromethylphenyl)deutoroporphyrin dimethylester (6f). Metalloporphyrin 1 (136 mg, $0.21 \mathrm{mmol})$ and boronic acid $\mathbf{3 f}(152 \mathrm{mg}, 0.8 \mathrm{mmol})$ gave $4 \mathbf{f}(82 \mathrm{mg}, 55 \%)$ as a redviolet solid, mp $191-194{ }^{\circ} \mathrm{C}$ (dec.) and $\mathbf{6 f}(74 \mathrm{mg}, 42 \%)$ as a red-violet solid, $\mathrm{mp} 201-206{ }^{\circ} \mathrm{C}$ (dec.).

4f. ${ }^{1} \mathrm{H}$ NMR (200 MHz, $\left.\mathrm{CDCl}_{3}\right): \delta 3.19-3.36\left(\mathrm{~m}, 4 \mathrm{H}, 13^{2} / 17^{2}-\mathrm{CH}_{2}\right), 3.54\left(\mathrm{~s}, 3 \mathrm{H}, \mathrm{CH}_{3, \text { pyr }}\right), 3.56$ (s, $3 \mathrm{H}, \mathrm{CH}_{3, \text { pyr }}$ ), 3.64, 3.66, 3.73, 3.76 (s, $12 \mathrm{H}, \mathrm{CO}_{2} \mathrm{CH}_{3}, \mathrm{CH}_{3, \text { pyr }}$ ), 4.28-4.40 (m, $4 \mathrm{H}, 13^{1} / 17^{1}$ $\mathrm{CH}_{2}$ ), 7.47-7.87 (m, $\left.1 \mathrm{H}, \mathrm{CH}_{\text {aryl }}\right), 7.95-8.16\left(\mathrm{~m}, 2 \mathrm{H}, \mathrm{CH}_{\text {aryl }}\right), 8.28-8.35$ (m, $\left.1 \mathrm{H}, \mathrm{CH}_{\text {aryl }}\right), 8.56$ (s, $\left.1 \mathrm{H}, 8 / 3-\mathrm{H}_{\beta-\text { pyr }}\right), 9.88,9.92,10.01,10.04$ (s, $\left.4 \mathrm{H}, \mathrm{H}_{\text {meso }}\right)$.

6f. ${ }^{1} \mathrm{H}$ NMR $\left(200 \mathrm{MHz}, \mathrm{CDCl}_{3}\right): \delta 2.93\left(\mathrm{~s}, 3 \mathrm{H}, \mathrm{CH}_{3, \text { pyr }}\right.$ ), $3.00\left(\mathrm{~s}, 3 \mathrm{H}, \mathrm{CH}_{3, \text { pyr }}\right.$ ), 3.26-3.39 (m, 4 $\mathrm{H}, 13^{2} / 17^{2}-\mathrm{CH}_{2}, \mathrm{CH}_{3, \text { pyr }}$ ), 3.60-3.77 (m, $9 \mathrm{H}, \mathrm{CO}_{2} \mathrm{CH}_{3}, \mathrm{CH}_{3, \text { pyr }}$ ), 4.39-4.55 (m, $4 \mathrm{H}, 13^{1} / 17^{1}-$ $\mathrm{CH}_{2}$ ), 7.47-8.87 (m, $\left.4 \mathrm{H}, \mathrm{CH}_{\text {aryl }}\right), 8.00-8.06$ (m, $\left.2 \mathrm{H}, \mathrm{CH}_{\text {ary }}\right), 8.38$ (s, $\left.1 \mathrm{H}, \mathrm{CH}_{\text {ary }}\right), 8.52$ (s, $1 \mathrm{H}$, $\left.\mathrm{CH}_{\text {ary }}\right), 9.89,10.08,10.17,10.26$ (s, $\left.4 \mathrm{H}, 8 / 3-\mathrm{H}_{3-\text { pyr }}, \mathrm{H}_{\text {meso }}\right)$.

Zinc(II) 8-(3-bromophenyl)deuteroporphyrin dimethylester (4g). From metalloporphyrin 1 (136 mg, $0.21 \mathrm{mmol})$ and boronic acid $\mathbf{3 g}(161 \mathrm{mg}, 0.8 \mathrm{mmol})$ was isolated $\mathbf{4 g}(32 \mathrm{mg}, 21 \%)$ as a red-violet solid, mp $192-197{ }^{\circ} \mathrm{C}$ (dec.). ${ }^{1} \mathrm{H}$ NMR (200 $\left.\mathrm{MHz}, \mathrm{CDCl}_{3}\right): \delta 3.15-3.43(\mathrm{~m}, 10 \mathrm{H}$, $\left.13^{2} / 17^{2}-\mathrm{CH}_{2}, \mathrm{CH}_{3, \mathrm{pyr}}\right), 3.47-3.77$ (m, $12 \mathrm{H}, \mathrm{CO}_{2} \mathrm{CH}_{3}, \mathrm{CH}_{3, \text { pyr }}$ ), 4.22-4.38 (m, $4 \mathrm{H}, 13^{1} / 17^{1}-\mathrm{CH}_{2}$ ), 7.67-7.83 (m, $\left.1 \mathrm{H}, \mathrm{CH}_{\text {aryl }}\right), 7.90-8.04\left(\mathrm{~m}, 2 \mathrm{H}, \mathrm{CH}_{\text {aryl }}\right), 8.38\left(\mathrm{~m}, 1 \mathrm{H}, \mathrm{CH}_{\text {aryl }}\right), 9.05(\mathrm{~s}, 1 \mathrm{H}, 8 / 3-$ $\mathrm{H}_{\beta-\mathrm{pyr}}$ ), 9.87-10.00 (m, $\left.4 \mathrm{H}, \mathrm{H}_{\text {meso }}\right)$. 
Zinc(II) 8-(4-bromophenyl)deuteroporphyrin dimethylester (4h). Metalloporphyrin 1 (136 mg, $0.21 \mathrm{mmol})$ boronic acid $\mathbf{3 h}(161 \mathrm{mg}, 0.8 \mathrm{mmol})$ yielded $\mathbf{4 h}(41 \mathrm{mg}, 27 \%)$ as a redviolet solid, mp $195-199{ }^{\circ} \mathrm{C}$ (dec.). ${ }^{1} \mathrm{H}$ NMR (200 MHz, $\left.\mathrm{CDCl}_{3}\right)$ : $\delta 3.16-3.55\left(\mathrm{~m}, 13 \mathrm{H}, 13^{2} / 17^{2}\right.$ $\mathrm{CH}_{2}, \mathrm{CH}_{3, \text { pyr }}$ ), 3.66-3.80 (m, $9 \mathrm{H}, \mathrm{CO}_{2} \mathrm{CH}_{3}, \mathrm{CH}_{3 \text {,pyr }}$ ), 4.13-4.29 (m, $4 \mathrm{H}, 13^{1} / 17^{1}-\mathrm{CH}_{2}$ ), 7.83-7.99 (m, $4 \mathrm{H}, \mathrm{CH}_{\text {aryl }}$ ), 9.02 (s, $1 \mathrm{H}, 8 / 3-\mathrm{H}_{\beta-\text { pyr }}$ ), 9.60-9.97 (m, $4 \mathrm{H}, \mathrm{H}_{\text {meso }}$ ).

Zinc(II) 8-(4-fluorophenyl)deuteroporphyrin dimethylester (4i) and zinc(II) 5,8-di-(4fluorophenyl)deuteroporphyrin dimethylester (6i). Metalloporphyrin 1 (136 mg, $0.21 \mathrm{mmol})$ and boronic acid $3 \mathbf{i}$ (112 $\mathrm{mg}, 0.8 \mathrm{mmol}$ afforded $4 \mathbf{i}$ (92 $\mathrm{mg}, 66 \%)$ as a red-violet solid, mp 192$197{ }^{\circ} \mathrm{C}$ (dec.) and $6 \mathbf{i}(67 \mathrm{mg}, 32 \%)$ as a red-violet solid, mp $207-212{ }^{\circ} \mathrm{C}$.

4i. ${ }^{1} \mathrm{H}$ NMR (200 MHz, $\left.\mathrm{CDCl}_{3}\right): \delta 3.31\left(\mathrm{t}, J=7.6 \mathrm{~Hz}, 4 \mathrm{H}, 13^{2} / 17^{2}-\mathrm{CH}_{2}\right), 3.59\left(\mathrm{~s}, 3 \mathrm{H}, \mathrm{CH}_{3, \text { pyr }}\right.$ ), 3.59 (s, $3 \mathrm{H}, \mathrm{CH}_{3, \text { pyr }}$ ), 3.62 (s, $3 \mathrm{H}, \mathrm{CH}_{3, \text { pyr }}$ ), 3.68, 3.72, 3.74 (s, $9 \mathrm{H}, \mathrm{CO}_{2} \mathrm{CH}_{3}, \mathrm{CH}_{3, \text { pyr }}$ ), 4.39 (q, 4 $\left.\mathrm{H}, J=7.6 \mathrm{~Hz}, 13^{1} / 17^{1}-\mathrm{CH}_{2}\right), 7.55-7.63\left(\mathrm{~m}, 2 \mathrm{H}, \mathrm{CH}_{\text {aryl }}\right), 8.06-8.12\left(\mathrm{~m}, 2 \mathrm{H}, \mathrm{CH}_{\text {aryl }}\right), 9.08(\mathrm{~s}, 1 \mathrm{H}$, 8/3-H $\left.\mathrm{H}_{\text {B-pyr }}\right), 9.94,9.99,10.00,10.10$ (s, $4 \mathrm{H}, \mathrm{H}_{\text {meso }}$ ).

6i. ${ }^{1} \mathrm{H}$ NMR (200 MHz, $\left.\mathrm{CDCl}_{3}\right): \delta 3.32\left(\mathrm{t}, J=7.3 \mathrm{~Hz}, 4 \mathrm{H}, 13^{2} / 17^{2}-\mathrm{CH}_{2}\right), 3.52\left(\mathrm{~s}, 3 \mathrm{H}, \mathrm{CH}_{3, \text { pyr }}\right.$ ), 3.57 (s, $3 \mathrm{H}, \mathrm{CH}_{3, \text { pyr }}$ ), 3.58 (s, $3 \mathrm{H}, \mathrm{CH}_{3, \text { pyr }}$ ), 3.65 (s, $\left.3 \mathrm{H}, \mathrm{CH}_{3, \text { pyr }}\right), 3.74$ (s, $\left.6 \mathrm{H}, \mathrm{CO}_{2} \mathrm{CH}_{3}\right), 4.33-$ $4.42\left(\mathrm{~m}, 4 \mathrm{H}, 13^{1} / 17^{1}-\mathrm{CH}_{2}\right), 7.55-7.64\left(\mathrm{~m}, 4 \mathrm{H}, \mathrm{CH}_{\text {aryl }}\right), 8.07-8.14\left(\mathrm{~m}, 4 \mathrm{H}, \mathrm{CH}_{\text {aryl }}\right), 9.94,10.01$, $10.04,10.10$ (s, 4 H, 8/3- $\mathrm{H}_{3-\mathrm{pyr}}, \mathrm{H}_{\text {meso }}$ ).

Zinc(II) 8-(4-vinylphenyl)deuteroporphyrin dimethylester (4j) and zinc(II) 5,8-di(4vinylphenyl)deuteroporphyrin dimethylester (6j). Metalloporphyrin 1 (136 mg, $0.21 \mathrm{mmol})$ and boronic acid $\mathbf{3 j}$ (118 $\mathrm{mg}, 0.8 \mathrm{mmol})$ yielded $\mathbf{4 j}$ (58 $\mathrm{mg}, 41 \%)$ as a brown-violet solid, $\mathrm{mp}$ 204-207 ${ }^{\circ} \mathrm{C}$ (dec.) and $\mathbf{6 j}(57 \mathrm{mg}, 35 \%)$ as a brown-violett solid, mp $221-226{ }^{\circ} \mathrm{C}$ (dec.).

4j. ${ }^{1} \mathrm{H}$ NMR $\left(200 \mathrm{MHz}, \mathrm{CDCl}_{3}\right): \delta 3.38\left(\mathrm{t}, J=7.5 \mathrm{~Hz}, 4 \mathrm{H}, 13^{2} / 17^{2}-\mathrm{CH}_{2}\right), 3.61-3.81(\mathrm{~m}, 18 \mathrm{H}$, $\mathrm{CO}_{2} \mathrm{CH}_{3}, \mathrm{CH}_{3, \text { pyr }}$ ), 4.46 (q, $\left.4 \mathrm{H}, J=7.5 \mathrm{~Hz}, 13^{1} / 17^{1}-\mathrm{CH}_{2}\right), 5.53$ (d, $1 \mathrm{H}, J=11.1 \mathrm{~Hz}, \mathrm{CH}_{2 \text {,vinyl }}$ ), $6.11\left(\mathrm{~d}, 1 \mathrm{H}, J=11.1 \mathrm{~Hz}, \mathrm{CH}_{2 \text {,vinyl }}\right), 7.02-7.16$ (m, $\left.1 \mathrm{H}, \mathrm{CH}_{\text {vinyl }}\right), 7.95$ (d, $2 \mathrm{H}, J=8.1 \mathrm{~Hz}, \mathrm{CH}_{\text {aryl }}$ ), 8.17 (d, $\left.2 \mathrm{H}, J=8.1 \mathrm{~Hz}, \mathrm{CH}_{\text {aryl }}\right), 9.14$ (s, $\left.1 \mathrm{H}, 8 / 3-\mathrm{H}_{\beta-\text { pyr }}\right), 10.07,10.08,10.12,10.24$ (s, 4 H, $\mathrm{H}_{\text {meso }}$ ).

6j. ${ }^{1} \mathrm{H}$ NMR $\left(200 \mathrm{MHz}, \mathrm{CDCl}_{3}\right): \delta 3.26-3.38\left(\mathrm{~m}, 4 \mathrm{H}, 13^{2} / 17^{2}-\mathrm{CH}_{2}\right), 3.55-3.81(\mathrm{~m}, 18 \mathrm{H}$, $\mathrm{CO}_{2} \mathrm{CH}_{3}, \mathrm{CH}_{3, \text { pyr }}$ ), 4.35-4.53 (m, $4 \mathrm{H}, 13^{1} / 17^{1}-\mathrm{CH}_{2}$ ), 5.53 (d, $2 \mathrm{H}, J=10.8 \mathrm{~Hz}, \mathrm{CH}_{2, \text { vinyl }}$ ), 6.12 (d, $2 \mathrm{H}, J=10.8 \mathrm{~Hz}, \mathrm{CH}_{2 \text {,vinyl }}$ ), 7.02-7.16 (m, $2 \mathrm{H}, \mathrm{CH}_{\text {vinyl }}$ ), 7.92-8.01 (m, 4 H, $\mathrm{CH}_{\text {aryl }}$ ), 8.14-8.21 (m, $\left.4 \mathrm{H}, \mathrm{CH}_{\text {aryl }}\right), 10.07,10.13,10.17,10.23$ (s, $\left.4 \mathrm{H}, \mathrm{H}_{\text {meso }}\right)$.

Zinc(II) 8-(4-methoxyphenyl)deuteroporphyrin dimethylester (4k). From the reaction of metalloporphyrin 1 (136 mg, $0.21 \mathrm{mmol})$ and boronic acid 3k (122 mg, $0.8 \mathrm{mmol})$ was isolated 4k (148 mg, 99\%) as a red-violet solid, mp 179-185 ${ }^{\circ} \mathrm{C}$ (dec.). ${ }^{1} \mathrm{H}$ NMR (200 MHz, $\left.\mathrm{CDCl}_{3}\right): \delta$ 3.33 (t, $\left.J=6.0 \mathrm{~Hz}, 4 \mathrm{H}, 13^{2} / 17^{2}-\mathrm{CH}_{2}\right), 3.55$ (s, $3 \mathrm{H}, \mathrm{CH}_{3 \text {,pyr }}$ ), 3.62 (s, $3 \mathrm{H}, \mathrm{CH}_{3, \text { pyr }}$ ), 3.66 (s, $3 \mathrm{H}$, $\mathrm{CH}_{3, \text { pyr }}$ ), 3.70, 3.72, 3.73 (s, $9 \mathrm{H}, \mathrm{CO}_{2} \mathrm{CH}_{3}, \mathrm{CH}_{3, \text { pyr }}$ ), 4.13 (s, $\left.3 \mathrm{H}, \mathrm{OCH}_{3}\right), 4.37-4.46$ (m, $4 \mathrm{H}$, $\left.13^{1} / 17^{1}-\mathrm{CH}_{2}\right), 7.43\left(\mathrm{~d}, 2 \mathrm{H}, J=7.4 \mathrm{~Hz}, \mathrm{CH}_{\text {ary }}\right), 8.11\left(\mathrm{~d}, 2 \mathrm{H}, J=7.4 \mathrm{~Hz}, \mathrm{CH}_{\text {aryl }}\right), 9.10$ (s, $1 \mathrm{H}, 8 / 3-$ $\left.\mathrm{H}_{\beta-p y r}\right), 10.03,10.06,10.14,10.18$ (s, $4 \mathrm{H}, \mathrm{H}_{\text {meso }}$ ).

Zinc(II) 3,8-di(4-vinylphenyl)deuteroporphyrin dimethylester (5a) and zinc(II) 3,5,8-tri(4vinylphenyl) deuteroporphyrin dimethylester (7a). Following the General Procedure the reaction of metalloporphyrin 2 (152 $\mathrm{mg}, 0.2 \mathrm{mmol})$ and boronic acid $\mathbf{3 j}$ (237 $\mathrm{mg}, 1.6 \mathrm{mmol})$ led 
to the isolation of $\mathbf{5 a}(57 \mathrm{mg}, 35 \%)$ as a red-violet solid, $\mathrm{mp} 217-223{ }^{\circ} \mathrm{C}$ (dec.) and of $7 \mathbf{a}(50 \mathrm{mg}$, $28 \%$ ) as a brown-violet solid, $\mathrm{mp}>250{ }^{\circ} \mathrm{C}$.

5a. $\delta 2.82\left(\mathrm{t}, J=7.7 \mathrm{~Hz}, 2 \mathrm{H}, 13^{2} / 17^{2}-\mathrm{CH}_{2}\right), 2.98\left(\mathrm{t}, J=7.7 \mathrm{~Hz}, 2 \mathrm{H}, 13^{2} / 17^{2}-\mathrm{CH}_{2}\right), 3.12(\mathrm{~s}, 3 \mathrm{H}$, $\mathrm{CH}_{3, \text { pyr }}$ ), 3.39 (s, $3 \mathrm{H}, \mathrm{CH}_{3, \text { pyr }}$ ), 3.41 (s, $3 \mathrm{H}, \mathrm{CH}_{3, \text { pyr }}$ ), 3.59, 3.61, 3.68 (s, $9 \mathrm{H}, \mathrm{CO}_{2} \mathrm{CH}_{3}, \mathrm{CH}_{3, \text { pyr }}$ ), 3.78-3.91 (m, $\left.2 \mathrm{H}, 13^{1} / 17^{1}-\mathrm{CH}_{2}\right), 4.00-4.11\left(\mathrm{~m}, 2 \mathrm{H}, 13^{1} / 17^{1}-\mathrm{CH}_{2}\right), 5.53-5.65(\mathrm{~m}, 2 \mathrm{H}$,

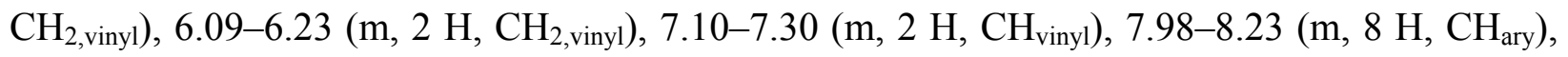
$8.69,8.87,9.68,9.72\left(\mathrm{~s}, 4 \mathrm{H}, \mathrm{H}_{\text {meso }}\right)$.

7a. $\delta 3.27-3.39\left(\mathrm{~m}, 4 \mathrm{H}, 13^{2} / 17^{2}-\mathrm{CH}_{2}\right), 3.54\left(\mathrm{~s}, 3 \mathrm{H}, \mathrm{CH}_{3, \mathrm{pyr}}\right), 3.60\left(\mathrm{~s}, 3 \mathrm{H}, \mathrm{CH}_{3, \mathrm{pyr}}\right), 3.66(\mathrm{~s}, 3 \mathrm{H}$, $\mathrm{CH}_{3, \text { pyr }}$ ), 3.71-3.73 (m, $\left.9 \mathrm{H}, \mathrm{CO}_{2} \mathrm{CH}_{3}, \mathrm{CH}_{3, \text { pyr }}\right), 4.33-4.59$ (m, $\left.4 \mathrm{H}, 13^{1} / 17^{1}-\mathrm{CH}_{2}\right), 5.14(\mathrm{~d}, 1 \mathrm{H}, \mathrm{J}$ $\left.=11.2 \mathrm{~Hz}, \mathrm{CH}_{2 \text {,vinyl }}\right), 5.51-5.65\left(\mathrm{~m}, 3 \mathrm{H}, \mathrm{CH}_{2 \text {,vinyl }}\right), 6.11\left(\mathrm{~d}, 1 \mathrm{H}, J=13.1 \mathrm{~Hz}, \mathrm{CH}_{2 \text {,vinyl }}\right.$, 6.59$6.64\left(\mathrm{~m}, 3 \mathrm{H}, \mathrm{CH}_{\text {vinyl }}, \mathrm{CH}_{\text {ary }}\right), 7.02-7.16\left(\mathrm{~m}, 3 \mathrm{H}, \mathrm{CH}_{\text {vinyl }}\right), 7.92-8.01$ (m, $\left.4 \mathrm{H}, \mathrm{CH}_{\text {ary }}\right), 8.13-8.20$ (m, $\left.4 \mathrm{H}, \mathrm{CH}_{\text {ary }}\right), 10.05,10.06,10.15$ (s, $\left.3 \mathrm{H}, \mathrm{H}_{\text {meso }}\right)$.

Zinc(II)-3,8-di(4-methoxyphenyl)-deuteroporphyrin dimethylester (5b) and zinc(II)-3,5,8tri(4-methoxyphenyl)-deuteroporphyrin dimethylester (7b). Similarly, from the reaction of metalloporphyrin 2 (152 mg, $0.2 \mathrm{mmol})$ and boronic acid $\mathbf{3 j}$ (244 mg, $1.6 \mathrm{mmol}$ ) were isolated 5b (110 mg, 68\%) as a red-violet solid, mp 208-213 ${ }^{\circ} \mathrm{C}$ (dec.) and $7 \mathbf{b}(34 \mathrm{mg}, 19 \%)$ as a redviolett solid, $\mathrm{mp} 234-238{ }^{\circ} \mathrm{C}$ (dec.).

5b. $\delta 2.85\left(\mathrm{t}, J=7.6 \mathrm{~Hz}, 2 \mathrm{H}, 13^{2} / 17^{2}-\mathrm{CH}_{2}\right), 2.99\left(\mathrm{t}, J=7.8 \mathrm{~Hz}, 2 \mathrm{H}, 13^{2} / 17^{2}-\mathrm{CH}_{2}\right), 3.17(\mathrm{~s}, 3 \mathrm{H}$, $\mathrm{CH}_{3, \text { pyr }}$ ), 3.42 (s, $3 \mathrm{H}, \mathrm{CH}_{3, \text { pyr }}$ ), 3.45 (s, $3 \mathrm{H}, \mathrm{CH}_{3, \text { pyr }}$ ), 3.56, 3.62, 3.67 (s, $9 \mathrm{H}, \mathrm{CO}_{2} \mathrm{CH}_{3}, \mathrm{CH}_{3, \text { pyr }}$ ), 3.98-4.35 (m, $\left.4 \mathrm{H}, 13^{1} / 17^{1}-\mathrm{CH}_{2}\right), 4.14\left(\mathrm{~s}, 3 \mathrm{H}, \mathrm{OCH}_{3}\right), 4.19\left(\mathrm{~s}, 3 \mathrm{H}, \mathrm{OCH}_{3}\right), 7.25-7.52(\mathrm{~m}, 4 \mathrm{H}$, $\left.\mathrm{CH}_{\text {ary }}\right), 7.99-8.20$ (m, $\left.4 \mathrm{H}, \mathrm{CH}_{\text {ary }}\right), 8.75,9.05,9.72,9.84$ (s, $4 \mathrm{H}, \mathrm{H}_{\text {meso }}$ ).

7b. $\delta 3.19-3.40\left(\mathrm{~m}, 4 \mathrm{H}, 13^{2} / 17^{2}-\mathrm{CH}_{2}\right), 3.56$ (s, $\left.3 \mathrm{H}, \mathrm{CH}_{3, \text { pyr }}\right), 3.60$ (s, $\left.3 \mathrm{H}, \mathrm{CH}_{3, \text { pyr }}\right), 3.68$ (s, $3 \mathrm{H}$, $\mathrm{CH}_{3, \text { pyr }}$ ), 3.72, 3.74, 3.78 (s, $9 \mathrm{H}, \mathrm{CO}_{2} \mathrm{CH}_{3}, \mathrm{CH}_{3, \text { pyr }}$ ), 3.33-4.53 (m, $\left.4 \mathrm{H}, 13^{1} / 17^{1}-\mathrm{CH}_{2}\right), 3.97$ (s, 3 $\left.\mathrm{H}, \mathrm{OCH}_{3}\right), 4.01\left(\mathrm{~s}, 3 \mathrm{H}, \mathrm{OCH}_{3}\right), 4.13\left(\mathrm{~s}, 3 \mathrm{H}, \mathrm{OCH}_{3}\right), 6.60-6.80\left(\mathrm{~m}, 4 \mathrm{H}, \mathrm{CH}_{\text {ary }}\right), 7.33-7.47(\mathrm{~m}, 4$ $\mathrm{H}, \mathrm{CH}_{\text {ary }}$ ), 8.01-8.16 (m, $\left.4 \mathrm{H}, \mathrm{CH}_{\text {ary }}\right), 10.06,10.15,10.19$ (s, $4 \mathrm{H}, \mathrm{H}_{\text {meso }}$ ).

\section{Acknowledgements}

We thank the Deutsche Forschungsgemeinschaft, the Fonds der Chemischen Industrie and the Grünenthal $\mathrm{GmbH}$ for their financial support.

\section{References}

1. Collman, J. P.; Wagenknecht; P. S.; Hutchison, J. E. Angew. Chem. 1994, 106, 1620; Angew. Chem., Int. Ed. 1994, 33, 1537.

2. Anderson, H. L.; Martin, S. J.; Bradley, D. C. Angew. Chem. 1994, 106, 710; Angew. Chem., Int. Ed. 1994, 33, 655.

3. Bonnett, R. Chem. Soc. Rev. 1995, 24, 19. 
4. Pandey, R. K.; Smith, K. M.; Dougherty, T. J. J. Med. Chem. 1990, 33, 2032.

5. Pandey, R. K.; Forsyth, T. P.; Smith, K. M. Tetrahedron Lett. 1992, 33, 5315.

6. Pandey, R. K.; Zheng, G.; Lee, D. A.; Dougherty, T. J.; Smith, K. M. J. Mol. Recog. 1996, 9, 118.

7. Suzuki, A.; Miyaura, N. Chem. Rev. 1995, 95, 2457.

8. (a) Chan, K. S.; Zhou, X.; Luo, B.; Mak, T. C. W. J. Chem. Soc., Chem. Commun. 1994, 271. (b) Zhou, X.; Zhou, Z.; Mak, T. C. W.; Chan, K. S. J. Chem. Soc., Perkin Trans 1 1994, 2519. (c) Chan, K. S.; Zhou, Y.; Au, M. T.; Tam, C. Y. Tetrahedron 1995, 51, 3129. (d) Zhou, X.; Tse, M. K.; Wan, T. S. M.; Chan, K. S. J. Org. Chem. 1996, 61, 3590.

9. An example for the synthesis of $\beta$-substituted porphyrin using the Heck reaction is described in: Ali, H.; van Lier, J. E. Tetrahedron 1994, 50, 11933.

10. Gauler, R.; Risch, N. Tetrahedron Lett. 1997, 38, 223.

11. Gauler, R.; Risch, N. Eur. J. Org. Chem. 1998, 1193.

12. Burrell, A. K.; Officer, D. L. Synlett 1998, 1297.

13. DiMagno, S. G.; Lin, V. S.-Y.; Therien, M. J. J. Org. Chem. 1993, 58, 5983. 